\title{
Killing Rate Kinetics of Commercially Available Brands of Ciprofloxacin and Cefotaxime on Clinical Bacterial Isolates Subjected to in vitro Antibiotic Treatments
}

Oke B. ${ }^{1}$, Iroha I.R. ${ }^{1}$, Moses I. B. ${ }^{1}$, Egwu I. H. ${ }^{1}$, Elom E. ${ }^{1}$, Uzoh C. V. ${ }^{2}$, Nwode V.F. ${ }^{2}$, Okpada J. $0 .{ }^{3}$, Agbom J. N. ${ }^{4}$, Peter I. U. ${ }^{1}$, Ibeka G. U. ${ }^{1}$ ${ }^{1}$ Department of Applied Microbiology, Ebonyi State University, Faculty of Sciences, Ebonyi State University, Abakaliki, Nigeria. ${ }^{2}$ Department of Biology/Microbiology/Biotechnology, Faculty of Science, Alex Ekwueme Federal University Ndufu-Alike Ikwo, Ebonyi State, Nigeria. ${ }^{3}$ Medical Laboratory Services, Alex Ekwueme Federal University Teaching Hospital, Abakaliki, Ebonyi State, Nigeria. ${ }^{4}$ Federal College of Agriculture, Ivo, Ebonyi State, Nigeria.

*Corresponding author's E-mail: ben_iyke70@yahoo.com

Received: 14-07-2020; Revised: 21-09-2020; Accepted: 28-09-2020; Published on: 20-10-2020.

\section{ABSTRACT}

The objective of this study was to determine the killing rate kinetics of commercially available brands of ciprofloxacin and cefotaxime on clinical isolates of Salmonella spp and Escherichia coli subjected to in vitro antibiotic treatments. One hundred and fifty (150) midstream urine samples were collected from urinary tract infection (UTI) patients attending Federal Teaching Hospital Abakaliki. Exactly 82 bacterial species ( $52 \mathrm{E}$. coli and 30 Salmonella species) were isolated from the urine samples after standard microbiological analysis. Time curve assay was done to determine the antibacterial activities of different company brands of ciprofloxacin and cefotaxime against Salmonella species and Escherichia coli using a sequential treatment scheme. Identified E. coli and Salmonella spp isolates were highly susceptible to meropenem, gentamicin, and ciprofloxacin but resistant to cefotaxime, ceftriaxone, and trimethoprim. Average MARI values of 0.4 and 0.2 were observed for $E$. coli and Salmonella spp respectively. Killing rate kinetics results showed that at 0 initial hour incubation without antibiotics, bacterial colonies were too numerous to count (TNTC). The growth of colonies when ciprofloxacin B and cefotaxime $E$ were used stops from the $3^{\text {rd }}$ hour till the $6^{\text {th }}$ hour. In contrast, growth was observed from the $3^{\text {rd }}$ hour till the $6^{\text {th }}$ hour in a decreasing order when cefotaxime $\mathrm{F}$ was used. Our findings showed that some local brands of ciprofloxacin and cefotaxime have low efficacy when compared to the antibiotics procured from Oxoid Company, UK

Keywords: Killing rate kinetics, E. coli, Salmonella, UTI, antibiotics

QUICK RESPONSE CODE $\rightarrow$

DOI:

10.47583/ijpsrr.2020.v64i02.015

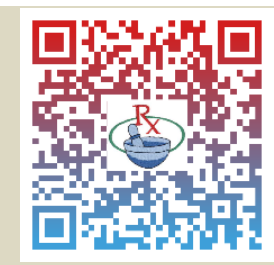

DOI link: $\underline{\text { http://dx.doi.org/10.47583/ijpsrr.2020.v64i02.015 }}$

\section{INTRODUCTION}

Sim equential and combination antibiotics therapies have been used to reduce not only the evolution of multidrug-resistant bacteria, but also the levels of antibiotics used in the treatment of bacterial infections. ${ }^{1}$ The decreased selection presence occurs when antibiotics are treated in appropriate order, which can prevent the emergence and spread of multidrug resistance. ${ }^{2}$ From the practical view point of antibiotics effectiveness, there is an important challenging question of whether the treatment history can cause potential carry-over effects on the additional antibiotic therapy.

The discovery of antimicrobial agents had a major impact on the rate of survival from infections. However, the changing patterns of antimicrobial resistance caused a demand for new antibacterial agents. Bacterial antimicrobial drug resistance is a worldwide emerging clinical and public health problem that is exacerbated by the diminishing number of new antimicrobial drugs in the pharmaceutical pipeline. ${ }^{3,45}$ However, drug resistance has also become a large and growing problem in infections that account for most of Africa's disease burden, including respiratory and diarrheal diseases. ${ }^{6,7}$ Much of the current discourse on infectious disease and drug resistance as it affects Sub-Saharan Africa is limited to the pressing problems associated with emerging- and re-emerging resistant organisms. Resistance, however, equally compromises the management of acute respiratory infections, sexually transmitted diseases and diseases spread by the fecal-oral route such as typhoid fever, cholera, dysentery and other diarrheal diseases. ${ }^{8}$

The effectiveness of currently available antibiotics is decreasing due to the increasing number of resistant strains causing infections. ${ }^{9}$ Resistance based on decreased entry of drugs has been found for penicillins, cephalosporin, aminoglycosides, and tetracyclines in the Enterobacteriaceae and Pseudomonas aeruginosa. Betalactamase resistance has increased significantly and is being encountered in Neisseria, Haemophilus, Enterobacteriaceae and Pseudomonas species..$^{5}$ Available therapeutic options for antibiotic-resistant organisms are severely limited, as these organisms frequently display a multidrug-resistant (MDR) phenotype. ${ }^{5,10,11}$ This study was designed to determine the killing rate kinetics of commercially available brands of ciprofloxacin and cefotaxime on clinical isolates of Salmonella spp and E. coli subjected to in vitro antibiotic treatments. 


\section{MATERIALS AND METHODS}

\section{Study Area}

This study was carried out in the Federal Teaching Hospital, Abakaliki (FETHA) Ebonyi State. Ebonyi state is located in the South East zone of Nigeria. It was created from Enugu and Abia State on Oct. $1^{\text {st }}, 1996$. The name Ebonyi was derived from its river called Ebonyi River and the state capital is Abakaliki. According 1996 population and Housing census, Ebonyi State is said to be made up of $1,064,156$ males and 1,112, 1791 females. There is an estimated population of 4.3 million people in EbonyiState. The land mass of Ebonyi State is $5,935 \mathrm{~km}^{2}$. Approximately $30 \%$ of the population of Ebonyi State dwell in urban areas and are civil servants and students. ${ }^{12}$ Federal Teaching Hospital is located at the centre of Abakaliki. It is made up of FETHA I and FETHA II.

\section{Sample Collection}

A total of one hundred and fifty (150) mid-stream urine samples were collected using sterile universal containers which have been labeled with the patients' information from the Federal Teaching Hospital Abakaliki and transported to the Applied Microbiology Laboratory complex of Ebonyi State University Abakaliki for bacteriological analysis.

\section{Microbiological analysis}

Urine samples were inoculated in nutrient broth for 24 hours for enrichment. Exactly $1 \mathrm{ml}$ of each urine samples was added to $9 \mathrm{ml}$ of nutrient broth for sufficient enrichment and incubated at $37^{\circ} \mathrm{C}$. The enriched urine samples were streaked onto Salmonella-Shigella (SSA) agar and cysteine lactose electrolyte deficient (CLED) agar respectively with the help of flame sterilized wire loop and incubated at $37{ }^{\circ} \mathrm{C}$ for 24 hours for recovery of Salmonella spp and E. coli isolates. The suspected Salmonella spp and $E$. coli isolates were further characterized using standard microbiology techniques such as Gram-staining, catalase test, motility test, and other biochemical tests which include indole test, citrate utilization test, oxidase test, methyl red test, Voges-Proskauer test, and sugar fermentation test. ${ }^{13}$

\section{Antibiotic Susceptibility Testing}

Antibiotic susceptibility test was done using the KirbyBauer disc diffusion method as described by the Clinical Laboratory Standards Institute (CLSI) guidelines. ${ }^{14}$ The following antibiotics were used for this study; ciprofloxacin
(5 $\mu \mathrm{g})$, meropenem (10 $\mu \mathrm{g})$, cefotaxime (30 $\mu \mathrm{g})$, chloramphenicol (30 $\mu \mathrm{g})$, nalidixic acid (30 $\mu \mathrm{g})$, sulphamethoxazole/trimethroprim (5 $\mathrm{gg}$ ), tetracycline (30 $\mu \mathrm{g})$, piperacillin $(10 \mu \mathrm{g})$, ceftriaxone $(30 \mu \mathrm{g})$, cefoxitin (30 $\mu \mathrm{g})$, ofloxacin $(5 \mu \mathrm{g})$, gentamicin $(10 \mu \mathrm{g})$, and norfloxacin $(10 \mu \mathrm{g})$. Mueller Hinton agar was prepared according to manufacturer's instructions and 0.5 McFarland equivalent standards of the test organisms was inoculated on the surface of the agar using a sterile cotton swab sticks. Test antibiotics disc listed above were aseptically placed on the inoculated agar plates and were incubated at $37{ }^{\circ} \mathrm{C}$ for 18 $24 \mathrm{hrs}$. Inhibition zone diameters were measured and results were interpreted as either susceptible or resistance based on Clinical Laboratory Standard Institute guidelines. ${ }^{14}$

\section{Determination of Multiple Antibiotic Resistance Index (MARI)}

The MARI value of the test isolates was evaluated to establish the standard antibiotic regimens for sequential in vitro treatments. The MARI was calculated using the formula $(\mathrm{MARI}=\mathrm{x} / \mathrm{b}$, where $\mathrm{x}=$ the total number of resistance recorded and $b=$ total number of antibiotics tested. ${ }^{15}$

\section{Dynamic Time Kill Curve Analysis}

The time curve assay was carried out to determine the antibacterial activities of different company brands of ciprofloxacin and cefotaxime against Salmonella spp and $E$. coli using a sequential treatment scheme. ${ }^{16}$ The initial population $\left(10^{5} \mathrm{cfu} / \mathrm{ml}\right)$ of Salmonella spp and E. coli of clinical origin was inoculated in nutrient broth without antibiotics and was incubated at $37^{\circ} \mathrm{C}$ for $8 \mathrm{hrs}$. This served as control. The different concentrations of ciprofloxacin and cefotaxime from 2 to $10 \mathrm{mg} / \mathrm{ml}$ were inoculated with Salmonella spp and E. coli for $1-8 \mathrm{hrs}$. Samples were collected from $0 \mathrm{hr}$, then at $1 \mathrm{hr}$ interval for $8 \mathrm{hrs}$, and pourplated on Mueller- Hinton agar. It was incubated at $37{ }^{\circ} \mathrm{C}$ for 24- $48 \mathrm{hrs}$ to enumerate viable cells using a colony counting machine.

Escherichia coli isolated from males between the age ranges of 1-17 years were susceptible to meropenem (100 $\%)$, gentamicin (85.7\%), ciprofloxacin (85.7\%) and ofloxacin $(57.1 \%)$, but exhibited resistance to cefotaxime (71.4\%), ceftriaxone (71.4\%), norfloxacin (57.1\%), trimethroprim (57.1\%), kanamycin (57.1\%), and tetracycline (57.1\%) (Table 3).

Table 1: Demographic Distribution of Samples According to Age and Sex of Patients

\begin{tabular}{|c|c|c|c|}
\hline Age (years) & \multicolumn{2}{|c|}{ Sex } & No. of Urine Samples collected \\
\hline 1-17 (children) & Males & Females & 45 \\
\hline 18-Above (adults) & 20 & 25 & 105 \\
\hline Total & 40 & 65 & $\mathbf{1 5 0}$ \\
\hline
\end{tabular}

A total of 82 bacterial species ( 52 E. coli and 30 Salmonella species) were isolated and identified from 150 mid-stream urine samples (Tables $2 a$ \& $2 b$ ). 
Table 2a: Frequency Distribution of Organisms Isolated from Male Urine Samples

\begin{tabular}{|c|c|c|c|}
\hline Age (Years) & No of urine Samples collected & No of $E$. coli & No of Salmonella species \\
\hline $1-17$ Children & 20 & $7(31.8 \%)$ & $5(33.3 \%)$ \\
\hline 18-Above (Adults) & 40 & $15(68.2 \%)$ & $10(66.7 \%)$ \\
\hline Total & 60 & $22(100 \%)$ & $15(100 \%)$ \\
\hline
\end{tabular}

Table 2b: Frequency Distribution of Organisms Isolated from female Urine Samples

\begin{tabular}{|c|c|c|c|}
\hline Age (Years) & No of urine Samples collected & No of $\boldsymbol{E}$. coli & No of Salmonella species \\
\hline 1 1-17 Children & 25 & $13(43.3 \%)$ & $8(53.3 \%)$ \\
\hline 18-Above (Adults) & 65 & $17(56.7 \%)$ & $7(46.7 \%)$ \\
\hline Total & 90 & $30(100 \%)$ & $15(100 \%)$ \\
\hline
\end{tabular}

Table 3: Susceptibility Profile of Escherichia coli isolates from male children (Age; 1-17)

\begin{tabular}{|c|c|c|c|c|c|}
\hline Antibiotics & Disc Potency & No Tested & No Sensitive (\%) & No Resistance (\%) & No Intermediate (\%) \\
\hline CTX & 30 & 7 & $1(14.3)$ & $5(71.4)$ & $1(14.3)$ \\
\hline NOR & 10 & 7 & $2(28.6)$ & $4(57.1)$ & $1(14.3)$ \\
\hline CRO & 30 & 7 & $1(14.3)$ & $5(71.4)$ & $1(14.3)$ \\
\hline SXT & 5 & 7 & $3(42.9)$ & $4(57.1)$ & $0(0.0)$ \\
\hline MEM & 10 & 7 & $7(100)$ & $0(0.0)$ & $0(0.0)$ \\
\hline NA & 30 & 7 & $3(42.9)$ & $3(42.9)$ & $1(14.3)$ \\
\hline K & 30 & 7 & $1(14.3)$ & $4(57.1)$ & $2(28.6)$ \\
\hline C & 30 & 7 & $1(14.3)$ & $3(42.9)$ & $3(42.9)$ \\
\hline FOX & 30 & 7 & $2(28.6)$ & $4(57.1)$ & $1(14.3)$ \\
\hline PRL & 10 & 7 & $4(57.1)$ & $2(28.6)$ & $1(14.3)$ \\
\hline TE & 30 & 7 & $3(42.9)$ & $4(57.1)$ & $0(0.0)$ \\
\hline CN & 10 & 7 & $6(85.7)$ & $1(14.3)$ & $0(0.0)$ \\
\hline CIP & 5 & 7 & $6(85.7)$ & $0(0.0)$ & $1(14.3)$ \\
\hline OFX & 5 & 7 & $4(57.1)$ & $1(14.3)$ & $2(28.6)$ \\
\hline
\end{tabular}

Key: Ciprofloxacin (CIP), meropenem (MEM), cefotaxime (CTX), chloramphenicol (C), kanamycin (K), nalidixic acid (NA), sulphamethoxazole/trimethroprim (SXT), tetracycline (TE), piperacillin (PRL), ceftriaxone (CRO), cefoxitin (FOX), ofloxacin (OFX), gentamicin (CN), and norfloxacin. (NOR). No = Number tested, Number Sensitive, Number Resistant

Escherichia coli isolated from females patients between the age range of $1-17$ years were susceptible to meropenem (84.6\%), gentamicin (76.9\%), ciprofloxacin (76.9\%) ofloxacin $84.6 \%$ ) and norfloxacin (76.9\%) but exhibited resistance to cefotaxime (92.3\%), ceftriaxone (61.5\%), trimethroprim (76.9\%), chloramphenicol (61.5\%) cefoxitin (76.9\%), and pipercilin (53.8\%) (Table 4).

Table 4: Susceptibility Profile of Escherichia coli isolates from female children (Age; 1-17 Yrs)

\begin{tabular}{|c|c|c|c|c|c|}
\hline Antibiotics & Disc Potency & No Tested & No Sensitive (\%) & No Resistance (\%) & No Intermediate (\%) \\
\hline CTX & 30 & 13 & $1(7.7)$ & $12(92.3)$ & $0(0.0)$ \\
\hline NOR & 10 & 13 & $10(76.9)$ & $3(23.1)$ & $0(0.0)$ \\
\hline SXT & 30 & 13 & $3(23.1)$ & $8(61.5)$ & $2(15.4)$ \\
\hline MEM & 5 & 13 & $3(23.1)$ & $10(76.9)$ & $0(0.0)$ \\
\hline NA & 10 & 13 & $11(84.6)$ & $0(0.0)$ & $2(15.4)$ \\
\hline K & 30 & 13 & $5(38.5)$ & $6(46.2)$ & $2(15.4)$ \\
\hline C & 30 & 13 & $4(30.8)$ & $6(46.2)$ & $3(23.1)$ \\
\hline FOX & 30 & 13 & $5(38.5)$ & $8(61.5)$ & $0(0.0)$ \\
\hline PRL & 30 & 13 & $2(15.4)$ & $10(76.9)$ & $1(7.7)$ \\
\hline TE & 10 & 13 & $4(30.8)$ & $7(53.8)$ & $2(15.4)$ \\
\hline CN & 30 & 13 & $6(46.2)$ & $6(46.2)$ & $1(7.7)$ \\
\hline CIP & 10 & 13 & $10(76.9)$ & $3(23.1)$ & $0(0.0)$ \\
\hline OFX & 5 & 13 & $10(76.9)$ & $2(15.4)$ & $1(7.7)$ \\
\hline
\end{tabular}

Key: Ciprofloxacin (CIP), meropenem (MEM), cefotaxime (CTX), chloramphenicol (C), kanamycin (K), nalidixic acid (NA), sulphamethoxazole/trimethroprim (SXT), tetracycline (TE), piperacillin (PRL), ceftriaxone (CRO), cefoxitin (FOX), ofloxacin (OFX), gentamicin (CN), and norfloxacin. (NOR) No = Number tested, Number Sensitive, Number Resistant 
Salmonella spp isolated from males between the age range of 1 - 17 years were susceptible to meropenem (100\%), gentamicin $(60 \%)$, ciprofloxacin $(60 \%)$, ofloxacin (60\%), cefoxitin (60\%), cefotaxime (60\%), nalidixic acid (60\%), ceftriaxone (60\%), trimethroprim (60\%), pipercilin (60\%), tetracycline (60\%), but exhibited resistance to norfloxacin (60\%) (Table 5).

Table 5: Susceptibility Profile of Salmonella species isolates from male Children (Age; 1- 17 years)

\begin{tabular}{|c|c|c|c|c|c|}
\hline Antibiotics & Disc Potency & No Tested & No Sensitive (\%) & No Resistance (\%) & No Intermediate (\%) \\
\hline CTX & 30 & 5 & $3(60)$ & $2(40)$ & $0(0.0)$ \\
\hline NOR & 10 & 5 & $1(20)$ & $3(60)$ & $1(20)$ \\
\hline CRO & 30 & 5 & $3(60)$ & $2(40)$ & $0(0.0)$ \\
\hline SXT & 5 & 5 & $3(60)$ & $2(40)$ & $0(0.0)$ \\
\hline MEM & 10 & 5 & $5(100)$ & $0(0.0)$ & $0(0.0)$ \\
\hline NA & 30 & 5 & $3(60)$ & $1(20)$ & $1(20)$ \\
\hline K & 30 & 5 & $1(20)$ & $2(40)$ & $2(40)$ \\
\hline C & 30 & 5 & $2(40)$ & $2(40)$ & $1(20)$ \\
\hline FOX & 30 & 5 & $3(60)$ & $0(0.0)$ & $2(40)$ \\
\hline PRL & 10 & 5 & $3(60)$ & $2(40)$ & $0(0.0)$ \\
\hline TE & 30 & 5 & $3(60)$ & $2(40)$ & $0(0.0)$ \\
\hline CN & 10 & 5 & $3(60)$ & $2(40)$ & $0(0.0)$ \\
\hline CIP & 5 & 5 & $3(60)$ & $1(20)$ & $1(20)$ \\
\hline OFX & 5 & 5 & $3(60)$ & $0(0.0)$ & $2(20)$ \\
\hline
\end{tabular}

Key: Ciprofloxacin (CIP), meropenem (MEM), cefotaxime (CTX), chloramphenicol (C), kanamycin (K), nalidixic acid (NA), sulphamethoxazole/trimethroprim (SXT), tetracycline (TE), piperacillin (PRL), ceftriaxone (CRO), cefoxitin (FOX), ofloxacin (OFX), gentamicin (CN), and norfloxacin. (NOR) No = Number tested, Number Sensitive, Number Resistant

Salmonella species isolated from females between the age ranges of 1-17 years were susceptible to meropenem (100 $\%)$, gentamicin (87.5\%), trimethroprim (87.5\%), and nalidixic acid (75 \%) but resistant to cefotaxime (75 \%), chloramphenicol (75\%), and tetracycline (63.5\%) (Table $6)$.

Table 6: Susceptibility Profile of Salmonella spp isolates from female Children (Age; 1-17)

\begin{tabular}{|c|c|c|c|c|c|}
\hline Antibiotics & Disc Potency & No Tested & No Sensitive (\%) & No Resistance (\%) & No Intermediate (\%) \\
\hline CTX & 30 & 8 & $1(12.5)$ & $6(75)$ & $1(12.5)$ \\
\hline NOR & 10 & 8 & $4(50)$ & $4(50)$ & $0(0.0)$ \\
\hline CRO & 30 & 8 & $1(12.5)$ & $2(25)$ & $5(62.5)$ \\
\hline SXT & 5 & 8 & $1(12.5)$ & $7(87.5)$ & $0(0.0)$ \\
\hline MEM & 10 & 8 & $8(100)$ & $0(0.0)$ & $0(0.0)$ \\
\hline KA & 30 & 8 & $6(75)$ & $0(0.0)$ & $2(25)$ \\
\hline C & 30 & 8 & $2(25)$ & $3(37.5)$ & $3(37.5)$ \\
\hline FOX & 30 & 8 & $2(25)$ & $6(75)$ & $0(0.0)$ \\
\hline PRL & 10 & 8 & $3(37.5)$ & $3(37.5)$ & $2(25)$ \\
\hline TE & 30 & 8 & $2(25)$ & $4(50)$ & $2(25)$ \\
\hline CN & 10 & 8 & $3(37.5)$ & $5(63.5)$ & $0(0.0)$ \\
\hline CIP & 5 & 8 & $7(87.5)$ & $1(12.5)$ & $0(0.0)$ \\
\hline OFX & 5 & 8 & $2(25)$ & $(0.0)$ & $6(75)$ \\
\hline
\end{tabular}

Key: Ciprofloxacin (CIP), meropenem (MEM), cefotaxime (CTX), chloramphenicol (C), kanamycin (K), nalidixic acid (NA), sulphamethoxazole/trimethroprim $(\mathrm{SXT})$, tetracycline (TE), piperacillin (PRL), ceftriaxone (CRO), cefoxitin (FOX), ofloxacin (OFX), gentamicin (CN), and norfloxacin (NOR), No = Number tested, Number Sensitive, Number Resistant

Escherichia coli isolated from male adults between the age ranges of 18 years and above was susceptible to meropenem (100\%), tetracycline (60\%), gentamicin (80
$\%)$, ofloxacin (80\%) and ciprofloxacin (66.7\%) but showed resistance to kanamycin (66.7\%) and nalidixic acid (53.3\%) (Table 7). 
Table 7: Susceptibility Profile of Escherichia coli Isolates from Male Adults (Age; 18-Above)

\begin{tabular}{|c|c|c|c|c|c|}
\hline Antibiotics & Disc Potency & No Tested & No Sensitive (\%) & No Resistance (\%) & No Intermediate (\%) \\
\hline CTX & 30 & 15 & $1(6.7)$ & $14(93.3)$ & $0(0.0)$ \\
\hline NOR & 10 & 15 & $6(40)$ & $6(40)$ & $3(20)$ \\
\hline CRO & 30 & 15 & $5(33.3)$ & $7(46.7)$ & $3(20)$ \\
\hline SXT & 5 & 15 & $6(40)$ & $6(40)$ & $3(20)$ \\
\hline MEM & 10 & 15 & $12(80)$ & $0(0.0)$ & $3(20)$ \\
\hline NA & 30 & 15 & $4(26.7)$ & $8(53.3)$ & $3(20)$ \\
\hline K & 30 & 15 & $1(6.7)$ & $10(66.7)$ & $4(26.7)$ \\
\hline C & 30 & 15 & $5(33.3)$ & $7(46.7)$ & $3(26.7)$ \\
\hline FOX & 30 & 15 & $3(20)$ & $7(46.7)$ & $5(33.3)$ \\
\hline PRL & 10 & 15 & $4(26.7)$ & $7(46.7)$ & $4(26.7)$ \\
\hline TE & 30 & 15 & $9(60)$ & $4(26.7)$ & $2(13.3)$ \\
\hline CN & 10 & 15 & $12(80)$ & $2(13.3)$ & $1(6.7)$ \\
\hline CIP & 5 & 15 & $10(66.7)$ & $3(20)$ & $2(13.3)$ \\
\hline OFX & 5 & 15 & $12(80)$ & $3(20)$ & $0(0.0)$ \\
\hline
\end{tabular}

Key: Ciprofloxacin (CIP), meropenem (MEM), cefotaxime (CTX), chloramphenicol (C), kanamycin (K), nalidixic acid (NA), sulphamethoxazole/trimethroprim (SXT), tetracycline (TE), piperacillin (PRL), ceftriaxone (CRO), cefoxitin (FOX), ofloxacin (OFX), gentamicin (CN), and norfloxacin. (NOR) No = Number tested, Number Sensitive, Number Resistant.

Escherichia coli isolated from male adults between the age ranges of 18 years and above were susceptible to meropenem (100\%), gentamicin (82.4\%), pipercilin (76.5 $\%)$, and ofloxacin (64.7\%) but resistant to ceftriaxone $(82.4$
$\%)$, cefotaxime (64.7\%), trimethroprim (64.7\%), nalidixic acid (58.8\%), kanamycin (82.4\%), chloramphenicol $(76.5$ $\%)$, tetracycline (52.9\%), and ciprofloxacin (52.9\%) (Table 8).

Table 8: Susceptibility Profile of E. coli Isolates from Female Adults (Age; 18 - Above)

\begin{tabular}{|c|c|c|c|c|c|}
\hline Antibiotics & Disc Potency & No Tested & No Sensitive (\%) & No Resistance (\%) & No Intermediate (\%) \\
\hline CTX & 30 & 17 & $0(0.0)$ & $11(64.7)$ & $6(35.3)$ \\
\hline NOR & 10 & 17 & $9(52.9)$ & $7(41.2)$ & $1(5.9)$ \\
\hline CRO & 30 & 17 & $0(0.0)$ & $14(82.4)$ & $3(17.6)$ \\
\hline MET & 5 & 17 & $3(17.6)$ & $11(64.7)$ & $3(17.6)$ \\
\hline NA & 10 & 17 & $17(100)$ & $0(0.0)$ & $0(0.0)$ \\
\hline K & 30 & 17 & $6(35.3)$ & $10(58.8)$ & $1(5.9)$ \\
\hline COX & 30 & 17 & $2(11.8)$ & $14(82.4)$ & $1(5.9)$ \\
\hline PRL & 30 & 17 & $2(11.8)$ & $13(76.5)$ & $2(11.8)$ \\
\hline TE & 30 & 17 & $7(41.2)$ & $8(47.1)$ & $2(11.8)$ \\
\hline CN & 10 & 17 & $13(76.5)$ & $0(0.0)$ & $4(23.5)$ \\
\hline CIP & 5 & 17 & $6(35.3)$ & $9(52.9)$ & $2(11.8)$ \\
\hline OFX & 5 & 17 & $14(82.4)$ & $0(0.0)$ & $3(17.6)$ \\
\hline
\end{tabular}

Key: Ciprofloxacin (CIP), meropenem (MEM), cefotaxime (CTX), chloramphenicol (C), kanamycin (K), nalidixic acid (NA), sulphamethoxazole/trimethroprim (SXT), tetracycline (TE), piperacillin (PRL), ceftriaxone (CRO), cefoxitin (FOX), ofloxacin (OFX), gentamicin (CN), and norfloxacin. (NOR) No = Number tested, Number Sensitive, Number Resistant

Salmonella species isolated from male adults between the age ranges of 18 years and above was susceptible to norfloxacin (60\%), ceftriaxone (70\%), meropenem (100
$\%)$, chloramphenicol (80\%), and gentamycin (70\%), but resistant to trimethroprim (90\%) kanamycin (80\%), and tetracycline (70\%) (Table 9 ) 
Table 9: Susceptibility Profile of Salmonella species isolates from male Adults (Age; 18 -Above)

\begin{tabular}{|c|c|c|c|c|c|}
\hline Antibiotics & Disc Potency & No Tested & No Sensitive (\%) & No Resistance (\%) & No Intermediate (\%) \\
\hline CTX & 30 & 10 & $4(40)$ & $4(40)$ & $2(20)$ \\
\hline NOR & 10 & 10 & $6(60)$ & $4(40)$ & $0(0)$ \\
\hline CRO & 30 & 10 & $7(70)$ & $2(20)$ & $1(10)$ \\
\hline SXT & 5 & 10 & $0(0)$ & $9(90)$ & $1(10)$ \\
\hline MEM & 10 & 10 & $10(100)$ & $0(0)$ & $0(0)$ \\
\hline NA & 30 & 10 & $3(30)$ & $5(50)$ & $2(20)$ \\
\hline K & 30 & 10 & $1(10)$ & $8(80)$ & $0(10)$ \\
\hline C & 30 & 10 & $8(80)$ & $2(20)$ & $3(30)$ \\
\hline FOX & 30 & 10 & $3(30)$ & $4(40)$ & $5(50)$ \\
\hline PRL & 10 & 10 & $2(20)$ & $3(30)$ & $1(10)$ \\
\hline TE & 30 & 10 & $2(20)$ & $7(70)$ & $2(20)$ \\
\hline CN & 10 & 10 & $7(70)$ & $1(10)$ & $6(60)$ \\
\hline CIP & 5 & 10 & $2(20)$ & $2(20)$ & $0(0)$ \\
\hline OFX & 5 & 10 & $5(50)$ & $5(50)$ & 0 \\
\hline
\end{tabular}

Key: Ciprofloxacin (CIP), meropenem (MEM), cefotaxime (CTX), chloramphenicol (C), kanamycin (K), nalidixic acid (NA), sulphamethoxazole/trimethroprim (SXT), tetracycline (TE), piperacillin (PRL), ceftriaxone (CRO), cefoxitin (FOX), ofloxacin (OFX), gentamicin (CN), and norfloxacin. (NOR) No = Number tested, Number Sensitive, Number Resistant

Salmonella spp isolated from female adults between the age range of 18 years and above was susceptible to cefotaxime (57.1\%) norfloxacin (85.7\%), meropenem
(85.7\%), nalidixic acid (71.2 \%), cefoxitin (57.1\%), pipercilin (85.7\%), gentamicin (100\%), and ofloxacin ( 85.7 \%) but resistant to trimethroprim (57.1\%) (Table 10$)$.

Table 10: Susceptibility Profile of Salmonella species isolates from female Adults (Age; 18-Above)

\begin{tabular}{|c|c|c|c|c|c|}
\hline Antibiotics & Disc Potency & No Tested & No Sensitive (\%) & No Resistance (\%) & No Intermediate (\%) \\
\hline CTX & 30 & 7 & $4(57.1)$ & $2(28.6)$ & $1(14.3)$ \\
\hline NOR & 10 & 7 & $6(85.7)$ & $1(14.3)$ & $0(0.0)$ \\
\hline CRO & 30 & 7 & $2(28.6)$ & $1(14.3)$ & $4(57.1)$ \\
\hline SXT & 5 & 7 & $3(42.9)$ & $4(57.1)$ & $0(0.0)$ \\
\hline MEM & 10 & 7 & $6(85.7)$ & $0(0.0)$ & $1(14.3)$ \\
\hline NA & 30 & 7 & $5(71.2)$ & $1(14.3)$ & $1(14.3)$ \\
\hline K & 30 & 7 & $2(28.6)$ & $3(42.9)$ & $2(28.6)$ \\
\hline C & 30 & 7 & $2(28.6)$ & $2(28.6)$ & $3(42.3)$ \\
\hline FOX & 30 & 7 & $4(57.1)$ & $3(43.3)$ & $0(0.0)$ \\
\hline PRL & 10 & 7 & $6(85.7)$ & $1(14.3)$ & $0(0.0)$ \\
\hline TE & 30 & 7 & $2(28.6)$ & $2(28.6)$ & $3(42.3)$ \\
\hline CN & 10 & 7 & $7(100)$ & $0(0.0)$ & $0(0.0)$ \\
\hline CIP & 5 & 7 & $2(28.6)$ & $2(28.6)$ & $3(42.3)$ \\
\hline OFX & 5 & 7 & $6(85.7)$ & $1(14.3)$ & $0(0.0)$ \\
\hline
\end{tabular}

Key: Ciprofloxacin (CIP), meropenem (MEM), cefotaxime (CTX), chloramphenicol (C), kanamycin (K), nalidixic acid (NA), sulphamethoxazole/trimethroprim $(\mathrm{SXT})$, tetracycline (TE), piperacillin (PRL), ceftriaxone (CRO), cefoxitin (FOX), ofloxacin (OFX), gentamicin (CN), and norfloxacin. (NOR) No = Number tested, Number Sensitive, Number Resistant

Table 11: Total and Average Multiple Antibiotics Resistance (MAR) Index for Escherichia coli.

\begin{tabular}{|c|c|c|}
\hline S/N & Bacterial isolates & MARI \\
\hline 1 & ECM 1-17 & 0.2 \\
\hline 2 & ECF 1-17 & 0.4 \\
\hline 3 & ECM 18-Above & 0.4 \\
\hline 4 & ECF 18-Above & 0.5 \\
\hline & Average MARI & $\mathbf{0 . 4}$ \\
\hline
\end{tabular}

Key: $\mathrm{ECM}=$ Escherichia coli males, $\mathrm{ECF}=$ Escherichia coli females
The result presented in Table 11 and 12 shows that average MARI values of 0.4 and 0.2 were observed for $E$. coli and Salmonella spp respectively.

A representative isolates from the samples were screened for time kill analysis using different company brands of ciprofloxacin and cefotaxime (Figures 1 to 8 ). 
Table 12: Total and Average Multiple Antibiotics Resistance (MARI) Index for Salmonella spp

\begin{tabular}{|c|c|c|}
\hline S/N & Bacterial isolates & MARI \\
\hline 1 & SM 1-17 & 0.1 \\
\hline 2 & SF 1-17 & 0.2 \\
\hline 3 & SM 18-Above & 0.3 \\
\hline 4 & SF 18-Above & 0.1 \\
\hline & Average MARI & $\mathbf{0 . 2}$ \\
\hline
\end{tabular}

Key: SM = Salmonella males, SF = Salmonella females
Results revealed that at zero (0) hour incubation without antibiotics, the colonies observed were too numerous to count (TNTC) across all the tests isolates. Meanwhile, the growth colonies on CIP B, and CTX E stopped from the $3^{\text {rd }}$ hour till the $6^{\text {th }}$ hour, when no growth was observed. For CTX F, from $3^{\text {rd }}$ to 8 hours, growth was found throughout the hours in a decreasing order. Generally, it was observed that all the company brands of the antibiotics showed no growth at the $6^{\text {th }}$ hour except CTX F. Results also revealed that the growth of all the isolates decreased with time among all the different antibiotics tested (Figures 1-8).

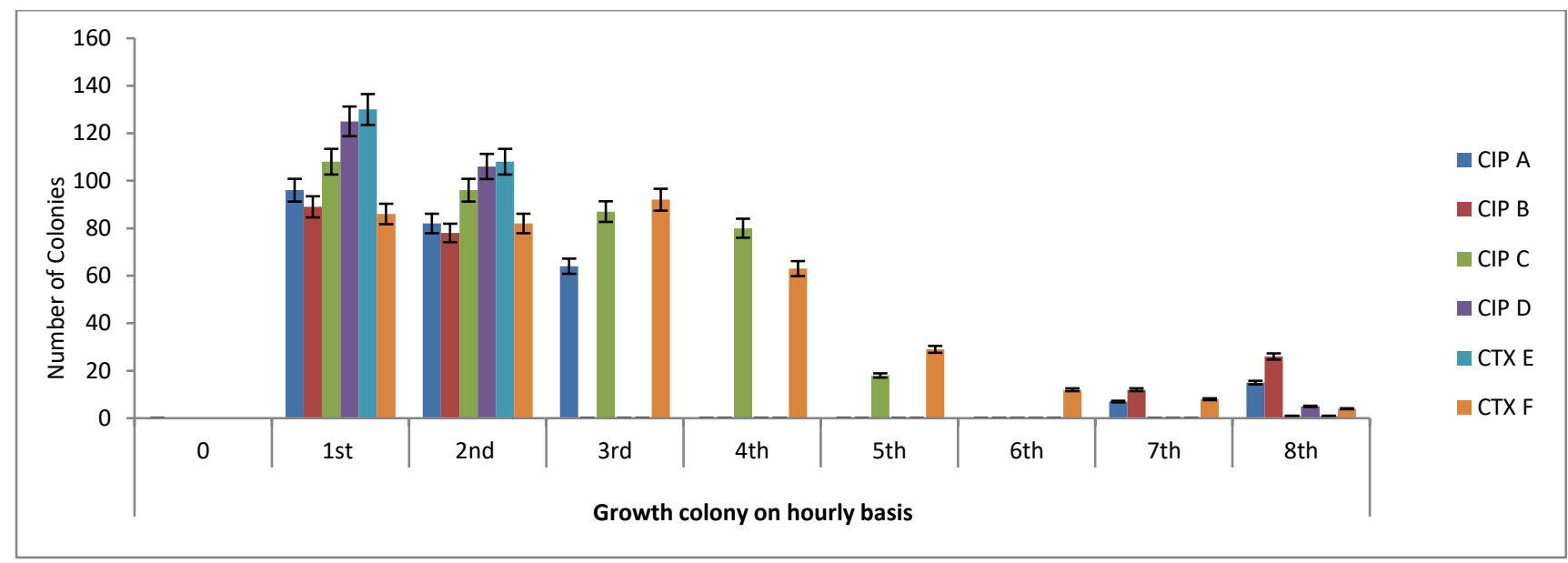

Figure 1: Different Company Brands of Antibiotics Tested Based on Hourly Interaction with the Organisms (Escherichia coli)

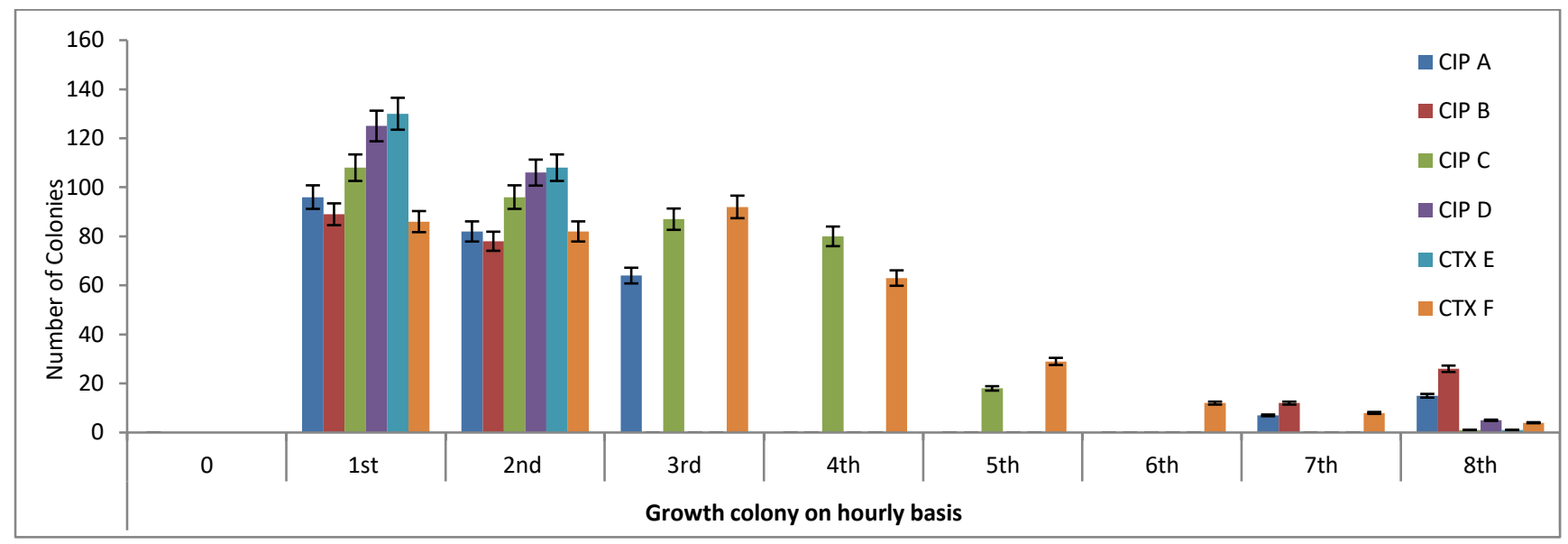

Figure 2: Different Company Brands of Antibiotics Tested Based on Hourly Interaction with the Organisms (Escherichia coli)

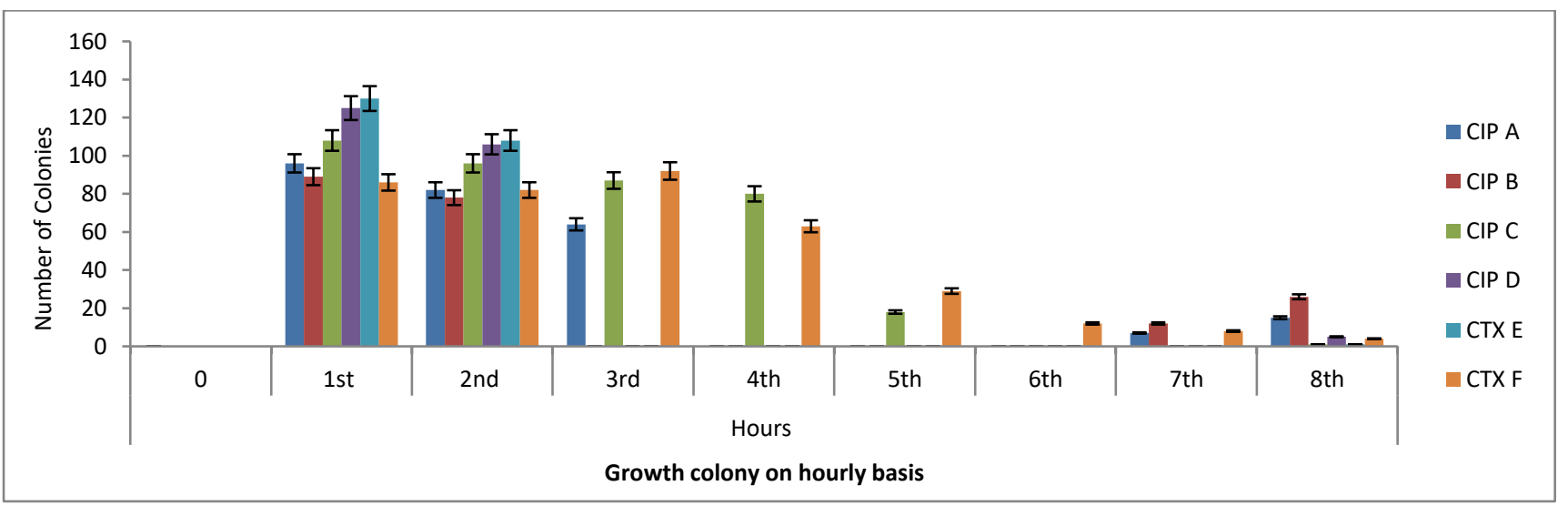

Figure 3: Different Company Brands of Antibiotics Tested Based on Hourly Interaction with the Organisms (Escherichia coli)

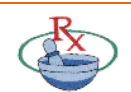




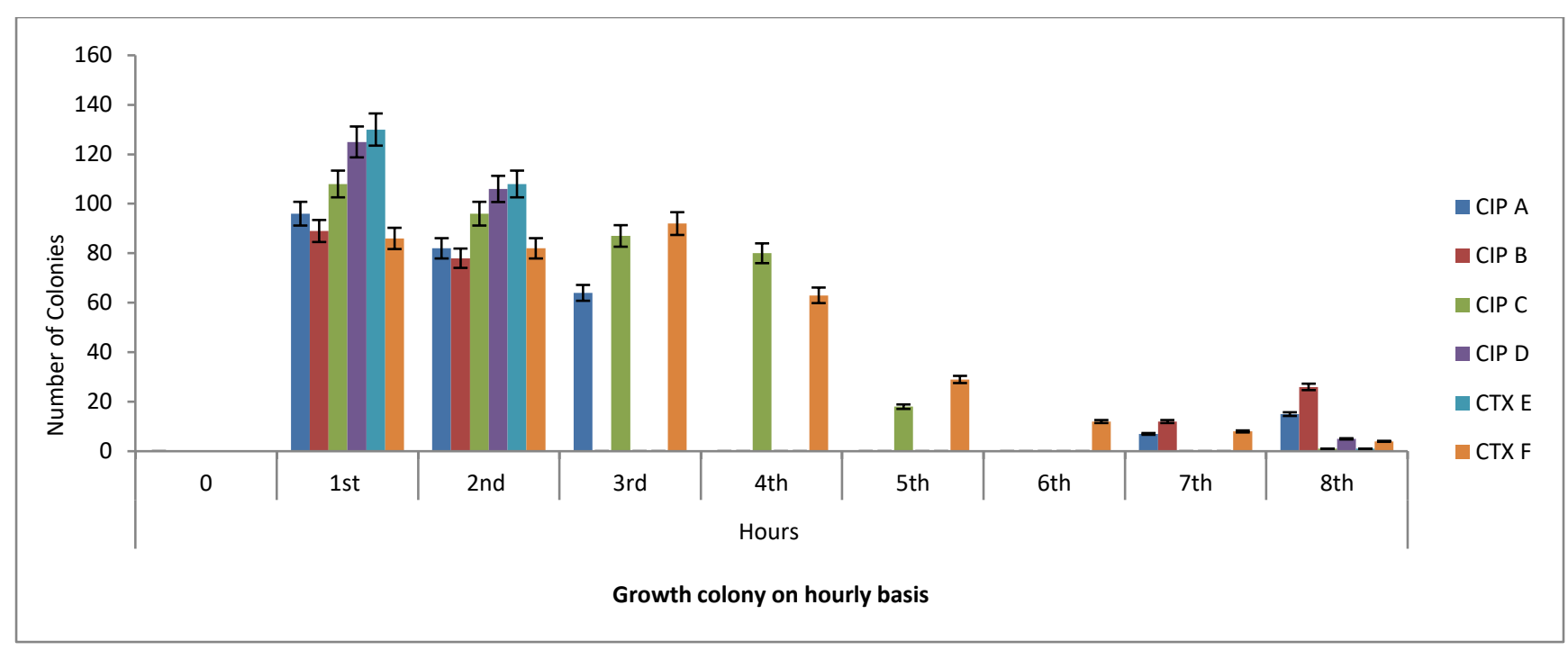

Figure 4: Different Company Brands of Antibiotics Tested Based on Hourly Interaction with the Organisms (Escherichia coli)

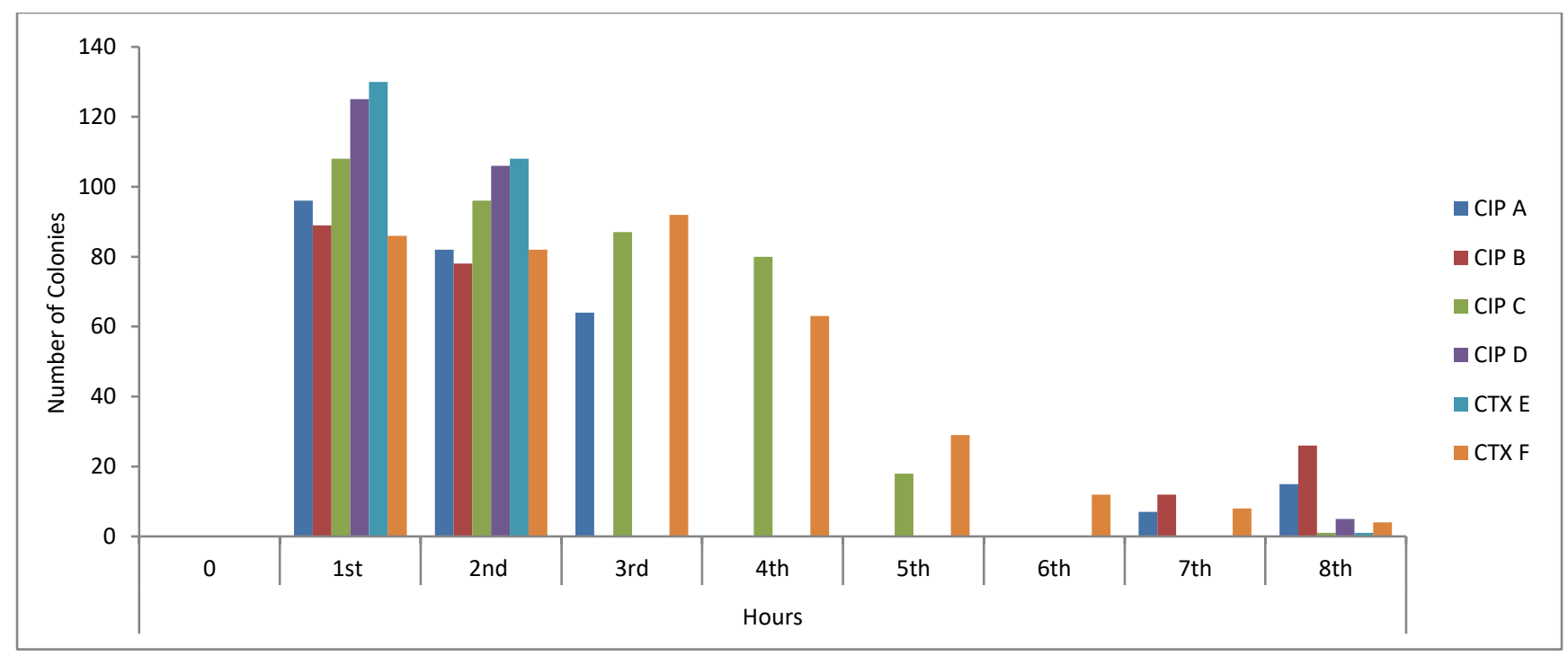

Figure 5: Different Company Brands of Antibiotics Tested Based on Hourly Interaction with the Organisms (Escherichia coli)

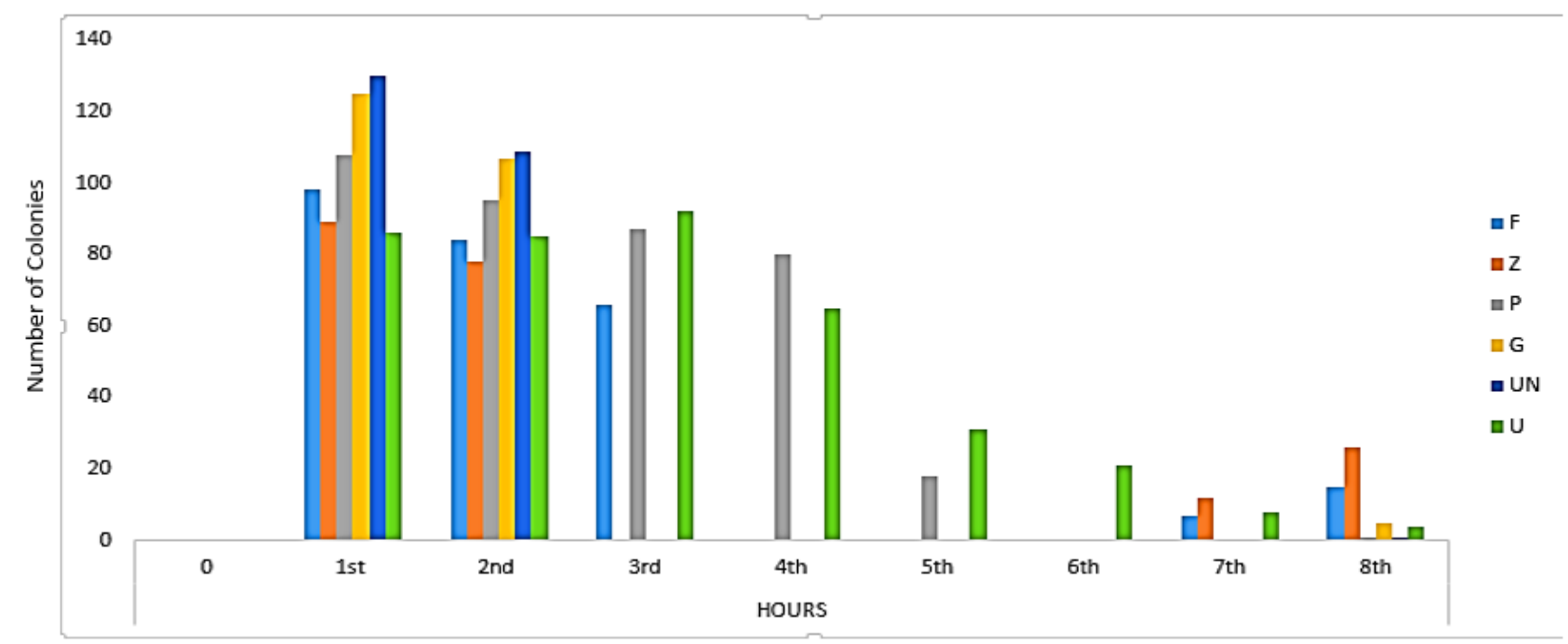

Figure 6: Different Company Brands of Antibiotics Tested Based on Hourly Interaction with the Organisms (Salmonella spp) 


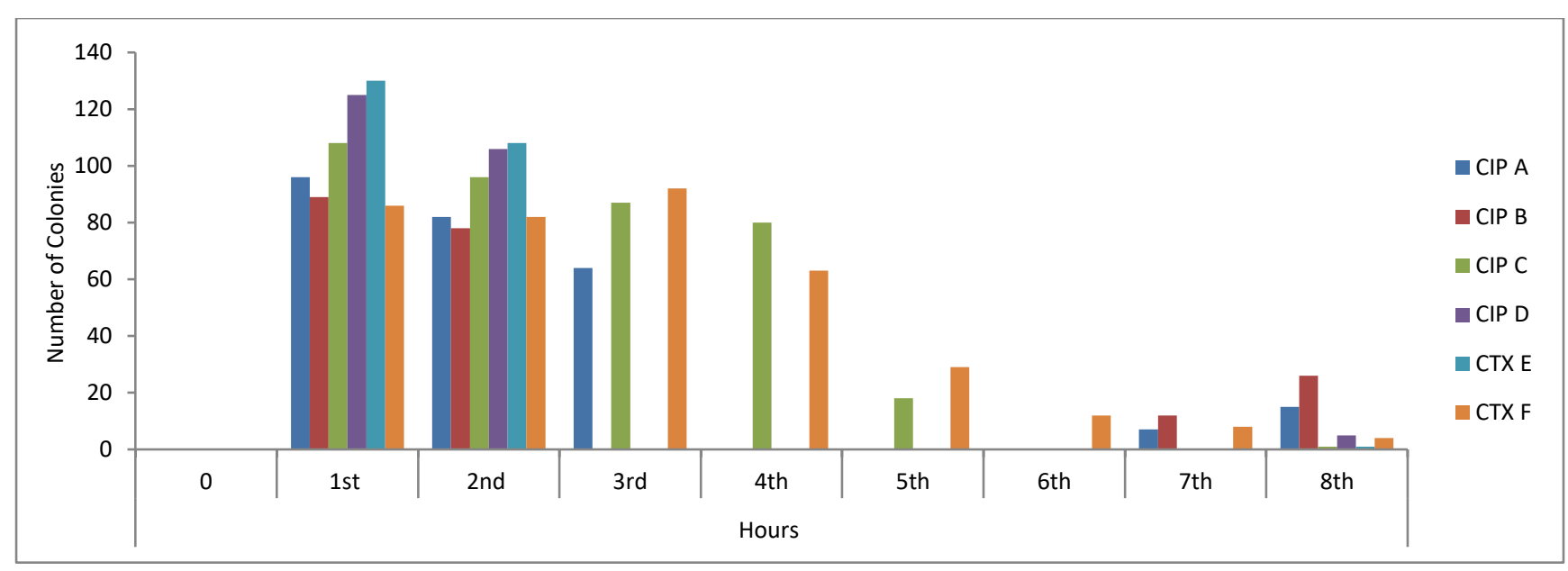

Figure 7: Different Company Brands of Antibiotics Tested Based on Hourly Interaction with the Organisms (Salmonella spp)

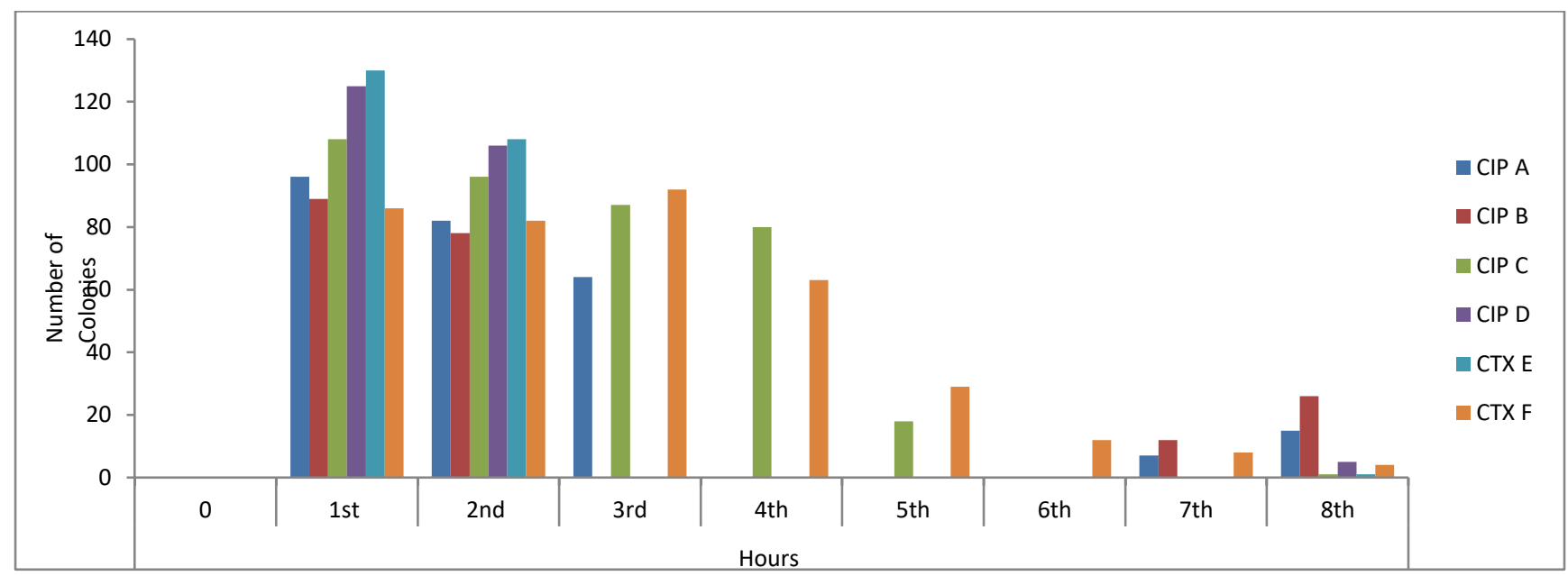

Figure 8: Different Company Brands of Antibiotics Tested Based on Hourly Interaction with the Organisms (Salmonella spp.)

\section{DISCUSSION}

The present study was carried out to determine the killing rate kinetics of commercially available brands of ciprofloxacin and cefotaxime on clinical isolates of Salmonella spp and E. coli subjected to in vitro antibiotics treatments. In our study, E. coli (52) and Salmonella spp (30) were all implicated in UTI among patients attending Federal Teaching Hospital Abakaliki. In a related study, Jombo et al. ${ }^{17}$ reported that the commonest bacteria recovered from UTI were Escherichia coli [18.6 \% (109)], Klebsiella pneumoniae [14.8\% (87)], Proteus species [13.1 $\%$ (77)], and Staphylococcus aureus [10.7 \% (63)]. The isolation of Escherichia coli and Salmonella species from the UTI patients in our study is in agreement with the findings of Iroha et al. ${ }^{18}$ who reported that 74 bacterial isolates were obtained from 80 urine samples collected. They reported that 30 (37.5\%) isolates were E. coli, 12 (15.0\%) were Pseudomonas aeruginosa, 11 (13.75\%) were Proteus species, 10 (12.5\%) were Staphylococcus aureus, 10 (12.5\%) were Klebsiella species while 1 (1.25\%) was Enterococcus feacalis. In another study, Bankole et al. ${ }^{19}$ reported that $E$. coli [128 (50.8\%)] were the most prevalent isolate out of a total of 252 bacterial isolates. In another study by Iroha et al. ${ }^{18} \mathrm{E}$ coli 30 (40.5 \%) was the predominant etiologic agent of pediatric UTI in FETHA; followed by Pseudomonas aeruginosa [12 (16.2 \%)], Proteus spp [11(14.9\%)], Klebsiella species [10 (13.5\%)], S. aureus [10 (13.5\%)], and Enterococcus feacalis [1(1.35\%)] being the least. The high frequency of $E$. coli observed in this study lends supports to the claim that $E$. coli is responsible for $>80 \%$ of all cases of urinary tract infection. ${ }^{20}$ There was no statistically significant difference in the frequency distribution of organisms when compared to the urine samples obtained $(p<0.05)$. It was observed that more female patients harboured more number of the causative agent of UTI. This result is in agreement with the findings of Nicolle ${ }^{21}$ who reported high prevalence of $E$. coli among females than their male counterparts. The predominance of infection (64\%) in females above 18 years old agreed with the report of Orrett and Shurland who reported differences in the level of infection among female and male counterparts with the females having the highest frequency of infection. ${ }^{22}$ The high prevalence of infection in females when compared to males is usually related to anatomical and pathogenic factors, e.g. the short length of the urethra hence, lesser distance of bacteria ascending up the tract, lack of antimicrobial properties of prostatic fluid as in males, hormonal changes affecting the adherence of bacteria to the mucosa, and 
urethra trauma during sexual intercourse. ${ }^{21}$ The latter factor accounts for the well-recognized "honeymoon cystitis" that is associated with UTI infections. Recent infection has also been found to be a predisposing factor to UTI acquisition. Other studies by Kibret ${ }^{23}$ also reported high frequency of UTIs among females, attributing it to the physiological and anatomical differences between males and females. However, with respect to the anatomical differences between females and males, the female urethra and the prostatic fluid in the vagina enhances the bacterial activities in the female urinary tract, thereby promoting the prevalence of the isolates. ${ }^{21}$ Also, the alteration in the microflora of the vagina allows the colonization of this isolates which can lead to urinary tract infection among females. ${ }^{21}$ On the contrary, the low incidence of occurrence of the isolates among males could be attributed to the environment in the urethra which reduces microbial growth. The longer distance between the anus and urethra meatus are all responsible for the low or reduced occurrence of the isolates among males. ${ }^{22}$ Escherichia coli were susceptible to meropenem (100\%), gentamicin (85.7\%), ciprofloxacin $(85.7 \%)$ and ofloxacin (57.1\%) while Salmonella species was susceptible to meropenem (100\%), gentamicin $(87.5$ $\%)$, trimethoprim (87.5\%), and nalidixic acid (75\%). However, Escherichia coli showed high frequency of resistance to cefotaxime $(71.4 \%)$, ceftriaxone $(71.4 \%)$, norfloxacin (57.1\%), trimethoprim (57.1\%), kanamycin (57.1\%), and tetracycline (57.1\%) while Salmonella species showed high frequency of resistance to cefotaxime (92.3\%), ceftriaxone (61.5\%), trimethoprim (76.9\%), chloramphenicol (61.5\%) cefoxitin (76.9\%), and piperacillin (53.8\%). The average MARI values of 0.4 and 0.2 were observed for $E$. coli and Salmonella spp respectively. High frequency of resistance to antibiotics as recorded in this present study poses a serious threat to public health. The antibiotic susceptibility patterns exhibited by the isolated E. coli and Salmonella spp in this study indicates the efficacy and potency of various antibiotics as every given antibiotic has a specific mode of action or interaction with different receptor sites of an organisms. This is in agreement with the study conducted in Ethiopia by Tesfaya et al. ${ }^{24}$ who reported comparable susceptibility rates to what we reported in our study. Our findings is in agreement with the work of Okonko et al. ${ }^{7}$ who also reported high resistance frequency of bacteria isolated from urine samples of patients attending Hospital for treatments. Our study also agrees with the findings of Afsharpaiman et al. ${ }^{25}$ who reported $E$. coli to have the least resistance to nalidixic acid in children. It also agrees with the findings of Badhan et al. ${ }^{26}$ who reported that nalidixic acid exhibited high resistance to all isolates especially the Gram-negative bacteria. Iroha et al. ${ }^{18}$ also reported that $E$. coli were completely (100\%) resistant to nalidixic acid. Our study agrees with the findings of Osundiya et al. ${ }^{27}$ who reported $94.2 \%$ susceptibility to meropenem by all the isolated bacterial pathogens. In another study, Iroha et al. ${ }^{18}$ reported that strains of $E$. coli were susceptible to 6 antibiotics, namely; nitrofurantoin (72\%), ciprofloxacin (81\%), amikacin (95\%), gentamicin (60\%), tobramycin (68
$\%)$, and ceftazidime (63\%). This is also in agreement with our findings. The results of the time killing rate kinetics showed that at zero (0) or initial hour incubation without antibiotics, the colonies observed were too numerous to count (TNTC) across all the tests isolates. Generally, it was observed that all the company brands of the antibiotics showed no growth at the $6^{\text {th }}$ hour except CTX F. The growth of all the isolates decreases with time among all the different antibiotics tested. This findings is similar to the study carried out by Iroha et al. ${ }^{16}$, who reported that the growth of organism decreases with time after 24 hours incubation.

\section{CONCLUSION}

This study implicated multi-drug resistant bacterial pathogens in UTI among patients in FETHA. E. coli was the most isolated causative agent in the urine samples analysed. Our Findings showed that meropenem was the most effective antibiotic against the bacterial pathogens isolated. The time killing analysis in our study suggests that commercially available antibiotics are less effective when compared to their manufacturers' claims. Production of more effective antibiotics is the way out in order to forestall the increasing frequency of antibiotic resistance being experienced nowadays. Sequential and combination antibiotics therapies could be used to reduce the evolution of multiple antibiotic-resistant bacteria, and also to stop the frequency of antibiotics use in the treatment of bacterial infections. This study augments efforts by other scientists to study the trends of pathogenic microorganisms' resistance patterns in various parts of the world. The high prevalence of E. coli observed in this present study and their frequency of resistance to conventional antibiotics call for concern to the general public. Proper adherence to dispensary ethics, strong public enlightenment strategies and production of more effective antibiotics is strongly recommended. Clinicians should strongly monitor patient's attitude to medications especially out patients. Strict laws against self-medication are important to forestall wrong dispensing especially by pharmaceutical stores

\section{REFERENCES}

1. Nichol D, Jeavons P, Fletcher AG, Bonomo RA, Maini PK, Paul $\mathrm{JL}$. Steering evolution with sequential therapy to prevent the emergence of bacterial antibiotic resistance. PLoS Comput Biol., 11(9), 2015, e1004493.

2. Warren, D. K, Hill, H. A, Merz, L. R, Kolle, M.H, Hayden, M.K, Fraser, V.J. Cycling empirical antimicrobial agents to prevent emergence of antimicrobial-resistant Gramnegative bacteria among intensive care unit patients. Crit Care Med, 32(12), 2004, 2450-6.

3. Spellberg, B., Powers, J.H., Brass, E.P., Miller, L.G. and Edwards, J.E. Trends in antimicrobial drug development: implications for the future. Clin. Infect. Dis., 38, 2004, 12791286.

4. Talbot, G.H., Bradley, J., Edwards, J.E., Gilbert, D., Scheld, M. and Bartlett, J.G. Bad bugs need drugs: an update on the development pipeline from the Antimicrobial Availability 
Task Force of the Infectious Diseases Society of America. Clin. Infect. Dis., 42, 2006, 657-668.

5. Okonko, I.O., Donbraye-Emmanuel, O.B., Ijandipe, L.A., Ogun, A.A., Adedeji, A.O. and Udeze, A.O. Antibiotics Sensitivity and Resistance Patterns of Uropathogens to Nitrofurantoin and Nalidixic Acid in Pregnant Women with Urinary Tract Infections in Ibadan, Nigeria. Middle East J. Sci. Res, 4(2), 2009a, 105-109.

6. Okeke, I.N., Aboderin, O.A, Byarugaba, D.K., Ojo, K.K. and Opintan, J.A. Growing problem of multidrug-resistant enteric pathogens in Africa. J. Med Sc., 4(2), 2007, 206-209.

7. Okonko, I.O., Soleye, F.A., Amusan, T.A., Ogun, A.A., Ogunnusi, T.A and Ejembi, J. Incidence of Multi-Drug Resistance (MDR) Organisms in Abeokuta, Southwestern Nigeria. Global J. Pharmacol. 3(2), 2009b, 69-80.

8. Okeke, I.NA., R. Laxminarayan, Z.A. Bhutta, A.G. Duse, P. Jenkins, T.F. O,Brien and A. Pablos-Mendez. Antimicrobial resistance in developing countries. Part I: recent trends and current status. Lancet Infectious Diseases, 5, 2005, 481-493.

9. Nawaz, S.K., Riaz, S. and Hasnain, S. Screening for antimethicillin resistant Staphylococcus aureus (MRSA) bacteriocin producing bacteria. Afr. J. Biotech., 8(3), 2009, 365-368.

10. Lewis, J. S., Herraera, M., Wickes, B., Patterson, J.E. and Jorgensen, J.H. First report of the emergence of CTX-M-type extended-spectrum _- lactamases (ESBLs) as the predominant ESBL isolated in a U.S. healthcare system. Antimicrob. Agents Chemother., 51, 2007, 4015-4021.

11. Chikere, C.B., Chikere, B.O. and Omoni, V.T. Antibiogram of clinical isolates from a hospital in Nigeria. Afr. J. Biotech., 7(24), 2008, 4359-4363.

12. The World Gazetteer. http//:www.world-gazetteer.com, 2014.

13. Cheesbrough, M. District Laboratory Practice in Tropical Countries. Part 2 Second Edition. Cambridge University press. New York, 2006, pp.12.

14. CLSI. 24th informational supplement. Clinical and Laboratory Standards Institute. M100-S24 Performance standards for antimicrobial susceptibility testing, 2014.

15. Moses I. B., Esimone C. O., Iroha I. R., Ugbo E. N., Orji J. O., Nwuzo A. C., Nwakaeze E. A., Agumah N. B., Emioye A. A., Ukpai E. G., Ogene L. N. First report on the antibiotic resistance profiles and virulence genes of Staphylococcus pseudintermedius colonizing dogs and humans in Abakaliki, South-East Nigeria. Research Journal of Microbiology, 15, 2020, 22-34.
16. Iroha, I. R., Ibezim, E. C., Ngwu, E. and Esimone, C. O. Evaluation of single and mixed kinetics of selected disinfectant against resistant and sensitive isolates of Pseudomonas aeruginosa and Staphylococcus aureus. Boce. Chim. Farma., 145(2), 2005, 1-9.

17. Jombo GTA, Emanghe UE, Amefule EN and Damen J.G. Urinary tract infections at a Nigerian university hospital: Causes, patterns and antimicrobial susceptibility profile. Journal of Microbiology and Antimicrobials, 3(6), 2011, 153159.

18. Iroha I, Afiukwa Ngozi, Nwuzo Agabus, Ejikeugwu Chika, Nwakaeze Emmanuel, Ukpai Ekenem, Anthonia Orji. Occurrence of ESBL-producing Escherichia coli and Klebsiella pneumoniae in urinary tract infection (UTI) patients. International Journal of Medicine and Pharmaceutical Research, 5(1), 2017, 59-65

19. Bankole O.Urinary tract infection in a rural community of Nigeria North. North American Journal of Medical Sciences, 3(2), 2017, 75-7.

20. Prescott, M. Harley, P. Klein, A. Chemotherapy. Microbiology $7^{\text {th }}$ Edition. McGraw - Hill, New. York. 25(4), 2005, 287-291.

21. Nicolle G. Recent developments in carbapenems. Expert Opin Investig Drug, 11(4), 2001, 529-44.

22. Orette FA and Shurland SM. The changing patterns of antimicrobial susceptibility of urinary pathogens in Trinidad. Singapore Journal Medicine, 39, 1998, 256-259.

23. Kibret M. Prevalence and antibiogram of bacterial isolates from urinary tract infections at Dessie Health Research Laboratory, Ethiopia. Asian Pac J Trop Biomed. 4, 2014, 1648.

24. Tesfaye A. Sero-prevalence of foot and mouth disease in cattle in Borena Zone, Oromia regional state, Ethiopia. Ethiopian Veterinary Journal, 20(1), 2009, 55-66.

25. Afshorpaiman, K., He, J., Ledesma, K., Hu, M., Tam, V. Pharmacokinetics and renal disposition of polymyxin $B$ in an animal model. Antimicrob. Agents Chemother., 56, 2012, 5724-5727.

26. Badhan R, Singh DV, Badhan LR, Kaur A. Evaluation of bacteriological profile and antibiotic sensitivity patterns in children with urinary tract infection: A prospective study from a tertiary care center. Indian J. Urol., 32, 2016, 50-6.

27. Osundiya, O. O., Oladele, R. O and Oduyebo, O. O. Multiple Antibiotic Resistance (Mar) Indices of Pseudomonas and Klebsiella species Isolates in Lagos University Teaching Hospital. African Journal of Clinical and Experimental Microbiology, 14(3), 2013, 7 - 9.

Source of Support: None declared.

Conflict of Interest: None declared.

For any question relates to this article, please reach us at: editor@globalresearchonline.net

New manuscripts for publication can be submitted at: submit@globalresearchonline.net and submit_ijpsrr@rediffmail.com 